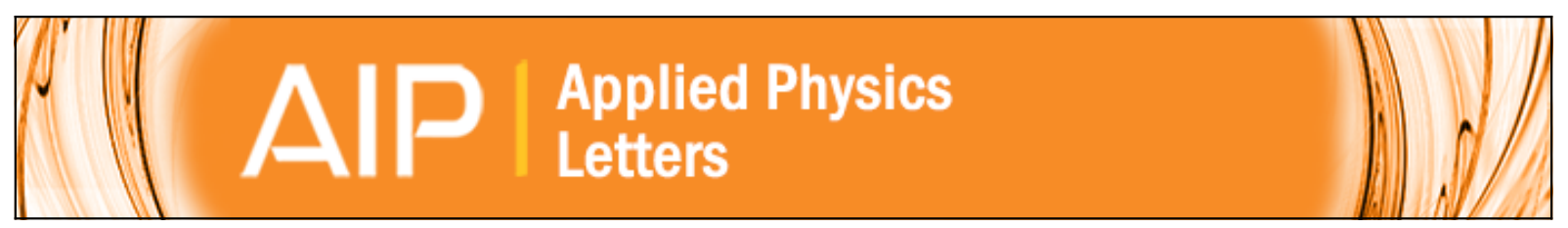

\title{
Photocurrent collection efficiency mapping of a silicon solar cell by a differential luminescence imaging technique
}

U. Rau, V. Huhn, L. Stoicescu, M. Schneemann, Y. Augarten, A. Gerber, and B. E. Pieters

Citation: Applied Physics Letters 105, 163507 (2014); doi: 10.1063/1.4898008

View online: http://dx.doi.org/10.1063/1.4898008

View Table of Contents: http://scitation.aip.org/content/aip/journal/apl/105/16?ver=pdfcov

Published by the AIP Publishing

\section{Articles you may be interested in}

Experimental demonstration of hot-carrier photo-current in an InGaAs quantum well solar cell

Appl. Phys. Lett. 104, 231115 (2014); 10.1063/1.4883648

Photoluminescence imaging for determining the spatially resolved implied open circuit voltage of silicon solar cells

J. Appl. Phys. 115, 044901 (2014); 10.1063/1.4862957

Voltage calibration of luminescence images of silicon solar cells

J. Appl. Phys. 115, 034508 (2014); 10.1063/1.4862400

Enhanced photocurrent and conversion efficiency in thin-film microcrystalline silicon solar cells using periodically textured back reflectors with hexagonal dimple arrays

Appl. Phys. Lett. 101, 173901 (2012); 10.1063/1.4761956

Contactless mapping of saturation currents of solar cells by photoluminescence

Appl. Phys. Lett. 100, 131108 (2012); 10.1063/1.3697704

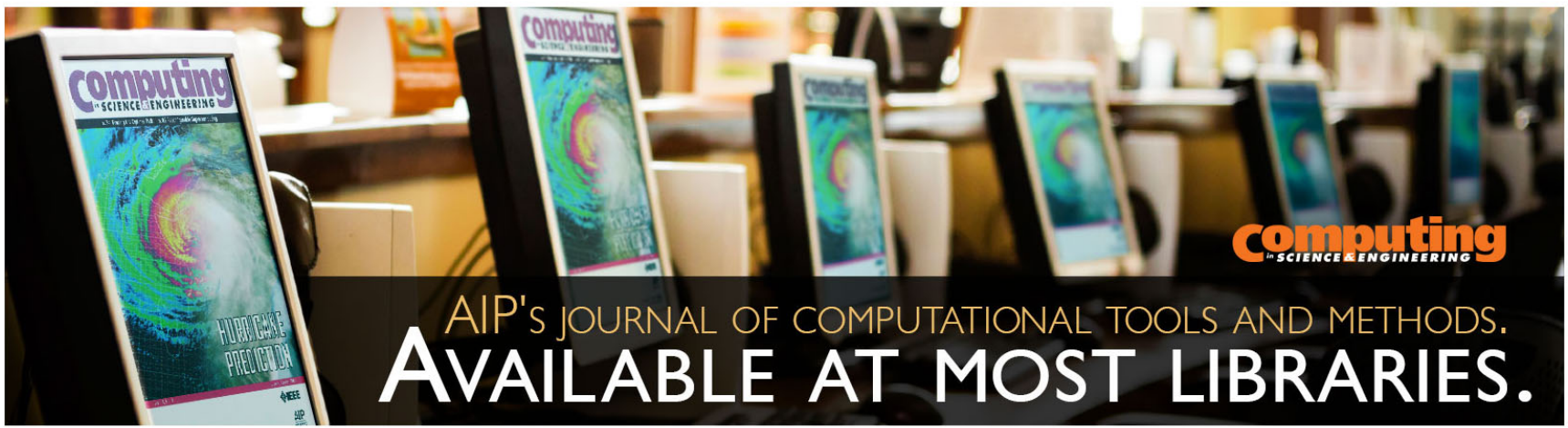




\title{
Photocurrent collection efficiency mapping of a silicon solar cell by a differential luminescence imaging technique
}

\author{
U. Rau, ${ }^{1, a)}$ V. Huhn, ${ }^{1}$ L. Stoicescu, ${ }^{2}$ M. Schneemann, ${ }^{1}$ Y. Augarten, ${ }^{1}$ A. Gerber, ${ }^{1}$ \\ and B. E. Pieters ${ }^{1}$ \\ ${ }^{1}$ IEK5-Photovoltaik, Forschungszentrum Jülich, 52425 Jülich, Germany \\ ${ }^{2}$ Institut für Photovoltaik, Universität Stuttgart, Pfaffenwaldring 47, 70569 Stuttgart, Germany
}

(Received 29 August 2014; accepted 1 October 2014; published online 21 October 2014)

\begin{abstract}
A differential electroluminescence imaging method for solar cells which yields local photocurrent collection efficiency maps is introduced. These maps attribute a value between zero and unity to each location on the cell. This value corresponds to the ratio between the current at the cell terminals and the locally generated photocurrent. The method is demonstrated for a multicrystalline silicon solar cell under constant illumination. If the point of maximum power output of the cell is chosen as the bias point, the method yields quantitative information on the local contribution to the maximum output power of the solar cell. (C) 2014 AIP Publishing LLC.
\end{abstract}

[http://dx.doi.org/10.1063/1.4898008]

During recent years, electroluminescence (EL) and photoluminescence (PL) imaging has become an important state-of-the-art characterization tool for solar cells and modules. $^{1-5}$ The different EL and/or PL based methods are fast, reliable, and quantitative. The basis for the quantitative evaluation of EL/PL experiments in solar cells is by given the reciprocity relation ${ }^{6,7}$ between electroluminescent emission and external photovoltaic quantum efficiency $Q_{\mathrm{e}}(E)$

$$
\begin{aligned}
\phi_{\mathrm{em}}(E) & =Q_{\mathrm{e}}(E) \phi_{\mathrm{bb}}(E)\left[\exp \left(\frac{V_{j}}{V_{t h}}\right)-1\right]+\phi_{\mathrm{sc}}(E) \\
& =\phi_{\mathrm{EL}}(E)+\phi_{\mathrm{PLsc}}(E)
\end{aligned}
$$

where $\phi_{\mathrm{bb}}(E)$ denotes the spectral photon flux density of a black body, $V_{\mathrm{j}}$ the voltage applied to the junction, and $V_{t h}$ the thermal voltage. In Eq. (1), $\phi_{\mathrm{em}}(E)$, the emitted photon flux density as a function of photon energy $E$, is a superposition of the pure EL emission $\phi_{\mathrm{EL}}$ stimulated by the junction voltage $V_{\mathrm{j}}$ and the short circuit (SC) emission $\phi_{\text {PLsc }}$ caused by the photoexcitation. ${ }^{7}$ For camera-based imaging methods, each pixel records a signal $S_{\text {cam }}(\mathbf{r})$ that depends on the local emission at a location $\mathbf{r}$ on the solar cell or module. This signal also depends on the quantum efficiency $Q_{\text {cam }}(E)$ of the camera $^{8,9}$ according to

$$
\begin{aligned}
S_{c a m}(\mathbf{r}) \approx & \int Q_{\mathrm{cam}}(E) Q_{\mathrm{e}}(E, \mathbf{r}) \phi_{\mathrm{bb}}(E) d E \exp \left(\frac{V_{j}(\mathbf{r})}{V_{t h}}\right) \\
& +\int Q_{\mathrm{cam}}(E) \phi_{\mathrm{sc}}(E, \mathbf{r}) d E \\
= & S_{0}(\mathbf{r}) \exp \left(\frac{V_{j}(\mathbf{r})}{V_{t h}}\right)+S_{P L s c}(\mathbf{r})=S_{E L}(\mathbf{r})+S_{P L s c}(\mathbf{r}),
\end{aligned}
$$

where we assume $V_{t h} \ll V_{j}$. The superposition of EL signal and the PL signal under short circuit condition underlying

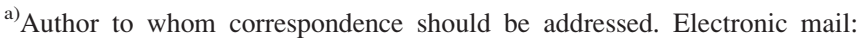
u.rau@fz-juelich.de
}

Eqs. (1) and (2) was derived explicitly in Ref. 7 from the principle of detailed balance. However, the same principle has been used implicitly earlier in Refs. 10-12 for the special case of silicon wafer cells. As pointed out by Bothe and Hinken, ${ }^{5}$ Eqs. (1) and (2) result from the linear superposition of the two solutions of the diffusion/recombination equation: (i) the solution for the dark minority carrier distribution and (ii) the solution for the distribution of photogenerated minority carriers under short circuit condition.

As can be seen from Eq. (2), an EL image depends on two local quantities; the local quantum efficiency $Q_{\mathrm{e}}(E, \mathbf{r})$, which results in the spatial dependence of the EL-prefactor $S_{0}(\mathbf{r})$, and the spatial distribution of the junction voltage $V_{j}(\mathbf{r})$. The external quantum efficiency $Q_{\mathrm{e}}(E, \mathbf{r})$ reflects a combination of local optical and recombination properties of the device, whereas variations of $V_{j}(\mathbf{r})$ result from the interplay of resistive and recombination effects within the network of resistors and recombination diodes making up the equivalent circuit of the spatially extended solar cell (see, e.g., Refs. 13 and 14). Furthermore, the signal is offset by the PL signal $S_{P L S c}(\mathbf{r})$ under short circuit conditions as well as by spurious reflections from the excitation light that are difficult to suppress. ${ }^{15,16}$ Due to these various influences, interpretation of EL/PL images is not straightforward and methods are required to separate the influence of each of these factors. Various methods have been proposed during the past years to isolate the consequences of the local series resistance ${ }^{10,17-19}$ or of the local diffusion length ${ }^{8}$ and reflectance losses ${ }^{20}$ from the other abovementioned effects.

The present paper uses differential, voltage driven luminescence images under constant illumination bias, which allow us to measure the device at any desired bias point, at the same time eliminating offsets that are not related to voltage variations. The present method yields an image of the photocurrent collection efficiency $f_{P C}$ as a function of the cell surface coordinate $\mathbf{r}$, by combined analysis of a small signal and a large signal differential measurement. The quantity $f_{P C}$ is defined as the ratio between the locally generated photocurrent $\delta I_{L}(\mathbf{r})$ and the resulting current $\delta I_{T}$ collected at the 
terminals of the cell. ${ }^{21}$ Due to this definition, we prefer the term 'photocurrent collection (PC) efficiency' and the symbol $f_{P C}$ over the term 'current transport efficiency' and the symbol $f_{T}$ used in Ref. 21.

The starting point of our approach is a reciprocity theorem derived by Wong and Green ${ }^{21}$ for a (linearized) equivalent network of a solar cell

$$
f_{P C}(\mathbf{r}):=\left.\frac{\delta I_{T}}{\delta I_{L}^{\mathbf{r}}}\right|_{\delta V_{T}=0}=\left.\frac{\delta V_{j}(\mathbf{r})}{\delta V_{T}}\right|_{\delta I_{L}=0} .
$$

Equation (3) states that is equivalent to measure $f_{P C}$ in either of two ways: (i) by generating a fixed differential photocurrent $\delta I_{L}^{\mathbf{r}}$ sequentially at all locations $\mathbf{r}$ and comparing it to the respective current $\delta I_{T}$ measured at the terminal without change of the terminal voltage, or (ii) by measuring the local junction voltage variation $\delta V_{j}(\mathbf{r})$ caused by a differential change of the terminal voltage $\delta V_{T}$ (without a change of the illumination). In the first case, the necessary number of measurements is defined by the desired spatial resolution, because a local excess generation of photocurrent together with the measurement of the corresponding change in terminal current is necessary to calculate $f_{P C}$ for each location separately. In contrast, because of Eq. (1), luminescence pictures yield access to the local junction voltage, thus replacing the cumbersome sequential direct measurement of $f_{P C}$ described above (with a separate determination of $\delta I_{T} / \delta I_{L}^{r}$ for each pixel) by a simple photographic procedure. To access arbitrary working conditions-including illumination bias, we need to apply a differential technique.

Let us first consider a large voltage difference to eclipse the effect of the (short-circuit) PL in Eq. (2). The difference signal between an external bias voltage $V_{T}=V_{b}$ and zero bias $\left(V_{T}=0\right)$ for the cell under constant illumination bias is given by

$$
\begin{aligned}
\Delta S_{\text {cam }}\left(\mathbf{r}, V_{b}\right) & =S_{c a m}\left(\mathbf{r}, V_{T}=V_{b}\right)-S_{c a m}\left(\mathbf{r}, V_{T}=0\right) \\
& =S_{0}(\mathbf{r}) \exp \left(\frac{V_{j}\left(\mathbf{r}, V_{b}\right)}{V_{t h}}\right) .
\end{aligned}
$$

Following Eq. (2), the first derivative of the camera signal with respect to the terminal voltage reads

$$
\frac{d}{d V_{T}} S_{c a m}\left(\mathbf{r}, V_{b}\right)=\frac{d V_{j}}{d V_{T}} \frac{S_{0}(\mathbf{r})}{V_{t h}} \exp \left(\frac{V_{j}\left(\mathbf{r}, V_{b}\right)}{V_{t h}}\right) .
$$

Considering a small variation $\delta V_{T}$ of the terminal voltage around the voltage bias $V_{T}=V_{b}$ for constant bias illumination, we arrive at the small signal difference

$$
\delta S_{c a m}\left(\mathbf{r}, V_{b}\right)=\frac{d V_{j}}{d V_{T}} \frac{S_{0}(\mathbf{r})}{V_{t h}} \exp \left(\frac{V_{j}\left(\mathbf{r}, V_{b}\right)}{V_{t h}}\right) \delta V_{T} .
$$

Combining the large signal with the small signal response, we arrive at

$$
f_{P C}(\mathbf{r})=\frac{d V_{j}}{d V_{T}}=\frac{\delta S_{c a m}\left(\mathbf{r}, V_{b}\right)}{\Delta S_{\text {cam }}\left(\mathbf{r}, V_{b}\right)} \frac{V_{t h}}{\delta V_{T}}
$$

which enables the determination of the photocurrent collection probability from the ratio of the large signal and the small signal response.

To experimentally verify our theoretical approach, we measured a $15.6 \mathrm{~cm} \times 15.6 \mathrm{~cm}$ 3-busbar industrial multicrystalline cell. The cell contact stage had a solid copper baseplate and two rows of contact pins along the outside two busbars. The centre pin in each row measured the cell voltage. The middle busbar was not contacted, so that largerscale series resistance variations could be observed. The cell's current voltage curve was measured, using this contacting setup, under standard test conditions (STC). For the luminescence imaging measurements, we used a setup from the Solarzentrum Stuttgart GmbH employing a Princeton Instruments Nirvana640 InGaAs camera $(640 \times 512$ pixel $)$ cooled to $-80^{\circ} \mathrm{C}$. Illumination was provided by a $90 \mathrm{~W}$, $808 \mathrm{~nm}$ diode laser, with an intensity adjusted such that the short circuit current of the cell under $808 \mathrm{~nm}$ was equivalent to the short circuit current under STC. This constant bias illumination was used for all measurements. Neither

(a)

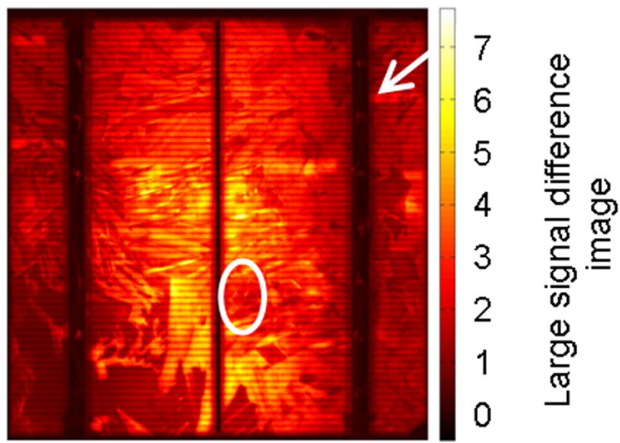

(b)

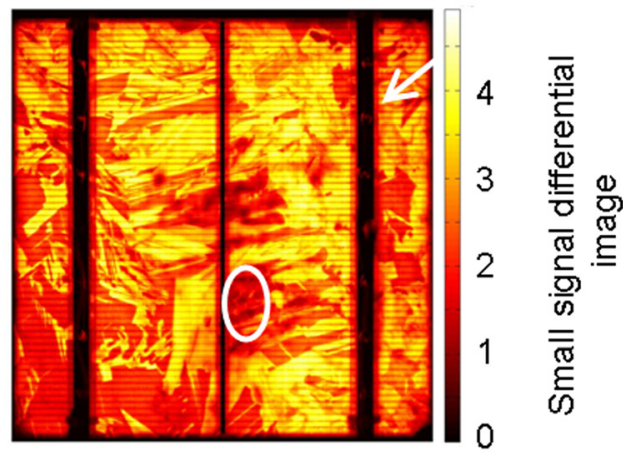

(c)

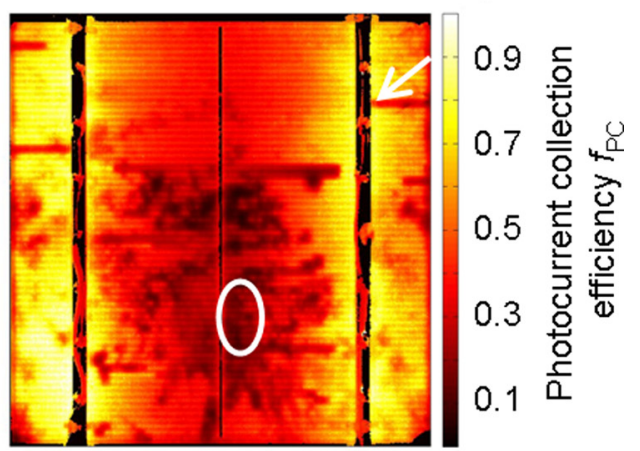

FIG. 1. (a) Large signal difference image of a polycrystalline Si solar cell (size $15.6 \times 15.6 \mathrm{~cm}^{2}$ ) obtained as the difference between a luminescence image taken at a terminal voltage of $V_{\mathrm{T}}=0.53 \mathrm{~V}$ and one taken at $V_{\mathrm{T}}=0 \mathrm{~V}$ under constant illumination bias. (b) Small signal differential image obtained as the difference between an image taken at a bias voltage of $V_{\mathrm{T}}=0.57 \mathrm{~V}$ and one taken at $V_{\mathrm{T}}=0.49 \mathrm{~V}$. (c) Photocurrent collection efficiency map obtained from dividing the data in (b) through the data in (a). 
background nor flatfield corrections was applied to the images, as they are accounted for in the analysis method.

Figures 1(a) and 1(b) show the large signal $\Delta S_{\text {cam }}(\mathbf{r})$ and the small signal differential image $\delta S_{\text {cam }}(\mathbf{r})$ of the multi-crystalline $\mathrm{Si}$ solar cell. The working point was defined by a bias voltage $V_{\mathrm{b}}=0.53 \mathrm{~V}$. The large signal difference picture was obtained by subtracting the luminescence image taken at zero voltage bias from that one obtained at $V_{\mathrm{b}}$. The small signal picture by subtracting the picture taken at $V_{T}=V_{b}-\delta V_{T} / 2$ from that at $V_{T}=$ $V_{b}+\delta V_{T} / 2$ with $\delta V_{T}=80 \mathrm{mV}$. All four images were taken under the same constant illumination intensity.

In Figs. 1(a) and 1(b), details such as different emission intensities caused by different grain orientations, reduced emission at grain boundaries, or dislocations, appear similarly, although some of the features appear more prominent in the small signal image. The differences between Figs. 1(a) and 1(b) are entirely explained by Eq. (7), as can be seen from the PC efficiency image (Fig. 1(c)), calculated from the ratio between the intensities displayed in Fig. 1(b) and Fig. 1(a). As predicted by Eq. (7), the PC map exhibits values between 0 and 1 , with $f_{P C}$ approaching unity at locations where perfect current collection is obtained, for instance close to the two contacted bus bars. Since the center bus bar was not contacted, $f_{P C}$ decreases towards the center of the cell. Especially, low values of $f_{P C}$ are caused by broken grid fingers (arrow for one example in Figs. 1(a)-1(c)) and regions of enhanced recombination (circle for one example in Figs. 1(a)-1(c)). It is interesting to note that in case of the broken finger (an example of collection losses due to a high local series resistance), the low PC values result from the relatively strong signal in the large signal picture (Fig. 1(a), arrow) which enters in the denominator of Eq. (7). This strong signal originates from the fact that the high series resistance due to the broken finger which implies that the local junction voltage $V_{j}(\mathbf{r})$ is much larger than the terminal voltage. The large value of $V_{j}(\mathbf{r})$ leads to a high large signal response $\Delta S_{\text {cam }}(\mathbf{r})$ [cf. Eq. (4)]. However, the high series resistance reduces the small signal response $\delta S_{\text {cam }}(\mathbf{r})$ with respect to $\Delta S_{\text {cam }}(\mathbf{r})$ by the poor ratio $d V_{j} / d V_{T}$ [cf. Eq. (5)]. In contrast, the low collection efficiency in the area of excessive recombination results from a low signal in the small signal picture (Fig. 1(b), circle) entering in the enumerator of Eq. (7). This effect is due to the reduced ratio of the local differential diode resistance in high recombination regions when compared to a normal series resistance. Therefore, the low $d V_{j} / d V_{T}$ value implies a reduced small signal $\delta S_{\text {cam }}(\mathbf{r})$, while the large signal image shows no peculiar reduction.

Due to the voltage dependence of the local diode resistances, the PC maps are expected to change with changing voltage bias. This is illustrated by the sequence of PC maps taken at bias voltages $V_{\mathrm{b}}=0.47 \mathrm{~V}$ (Fig. 2(a), maximum power point), $V_{\mathrm{b}}=0.51 \mathrm{~V}$ (Fig. 2 (b)), and $V_{\mathrm{b}}=0.53 \mathrm{~V}$ (Fig. 2(c), same as Fig. 1(c)). It is clearly seen from the figures that $f_{P C}$ decreases at any point with increasing $V_{\mathrm{b}}$. Even in the defect free regions in the upper center of the cell, the collection efficiency decreases due to the increasing ratio between series resistance and local diode resistance.

The PC map at the maximum power point (Fig. 2(a)) is especially valuable because here the differential (a)

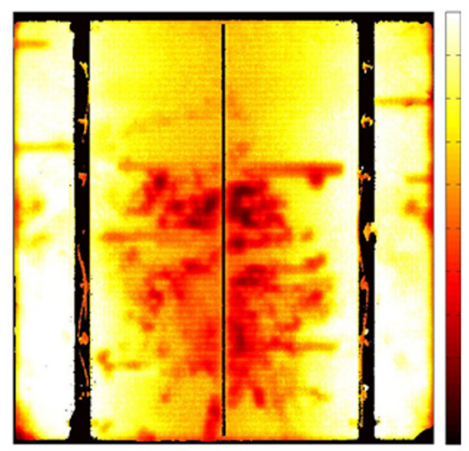

0.9

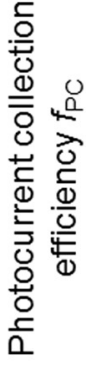

(b)

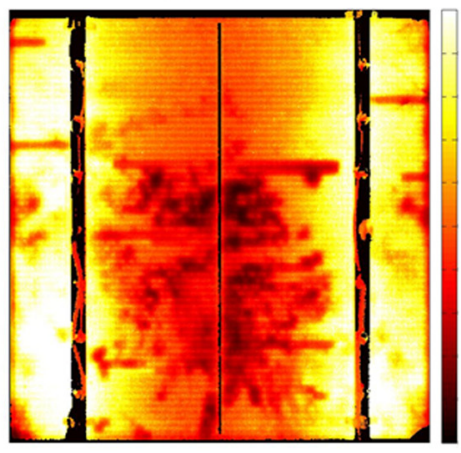

0.9

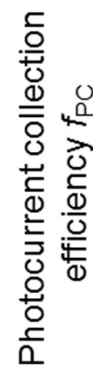

(c)
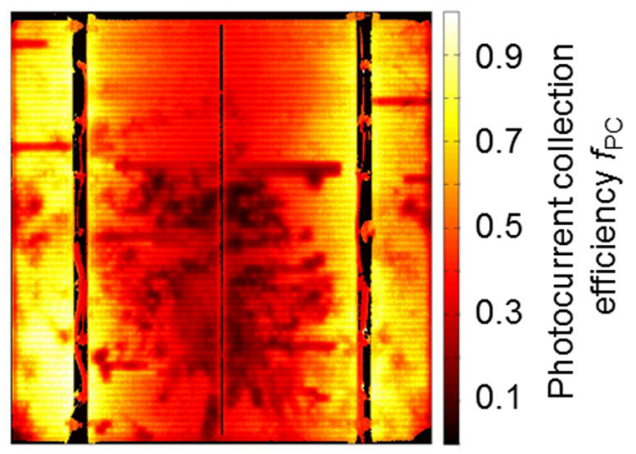

FIG. 2. Photocurrent collection efficiency maps measured at bias voltages $V_{\mathrm{b}}=0.47 \mathrm{~V}(\mathrm{a}), V_{\mathrm{b}}=0.51 \mathrm{~V}(\mathrm{~b})$, and $V_{\mathrm{b}}=0.53 \mathrm{~V}((\mathrm{c})$ - same as Fig. 1(c)).

contributions to the overall photocurrent from each point on the solar cell at the same time represent the differential contribution to the maximum output power. At the maximum power point, variations of the terminal current $\delta I_{T}$ lead to variations $\delta P_{\max }$ of the maximum output power according to $\delta P_{\max }=V_{T, m p p} \delta I_{T}{ }^{22}$ With the definition of the PC efficiency, we have

$$
\delta P_{\max }=V_{T, m p p} \frac{d I_{T}}{d I_{L}^{\mathbf{r}}} \delta I_{L}^{\mathbf{r}}=V_{T, m p p} f_{P C}(\mathbf{r}) \delta I_{L}^{\mathbf{r}},
$$

i.e., the PC map multiplied with the terminal voltage $V_{T, m p p}$ at the maximum power point yields the local contribution to the maximum output power $\delta P_{\max }$ per unit locally generated photocurrent $\delta I_{L}^{\mathrm{r}}$. As can be seen from Fig. 2(a), major losses to $\delta P_{\max }$ occur at the high recombination areas in the center of the cell with very low values of $f_{P C}$ in the range of $0.1-0.2$. Broken grid fingers lead to values of $f_{P C}$ of approximately 0.5 , whereas in the defect free regions close to the two contacted bus bars, $f_{P C}$ is very close to unity.

In summary, we have introduced a method to generate PC maps from differential EL images under illumination, i.e., under relevant working conditions. These PC maps quantitatively measure the current collection efficiency, and 
so the image yields comprehensive and quantitative information on the differential contribution of each area to the photocurrent at the maximum power point and, consequently, the differential contribution to the output power of the solar cell.

Financial support of the present work by the NRW-Ziel 2 project InnoPV (AZ 64.65.69-EN-1022B) and by the German Ministry of Environment (BMU) under Contract No. 0325364 is acknowledged.

${ }^{1}$ T. Fuyuki, H. Kondo, T. Yamazaki, Y. Takahashi, and Y. Uraoka, Appl. Phys. Lett. 86, 262108 (2005).

${ }^{2}$ T. Trupke, R. A. Bardos, M. C. Schubert, and W. Warta, Appl. Phys. Lett. 89, 044107 (2006).

${ }^{3}$ K. Ramspeck, K. Bothe, D. Hinken, B. Fischer, J. Schmidt, and R. Brendel, Appl. Phys. Lett. 90, 153502 (2007).

${ }^{4}$ T. Kirchartz, A. Helbig, B. E. Pieters, and U. Rau, "Electroluminescence analysis of thin-film solar modules," in Advanced Characterization Techniques for Thin Film Solar Cells edited by D. Abou-Ras, T. Kirchartz, and U. Rau (Wiley-VCH, Weinheim, Germany, 2011), pp 61-80.

${ }^{5} \mathrm{~K}$. Bothe and D. Hinken, "Quantitative luminescence characterization of crystallinesilicon solar cells," in Semiconductors and Semimetals, Advances in Photovoltaics Vol. 2, edited by G. P. Willeke and E. R. Weber (Elsevier, 2013), Vol. 89, pp. 259-339.

${ }^{6}$ U. Rau, Phys. Rev. B 76, 085303 (2007).

${ }^{7}$ U. Rau, IEEE J. Photovoltaics 2, 169 (2012).
${ }^{8}$ P. Würfel, T. Trupke, T. Puzzer, E. Schäffer, W. Warta, and S. W. Glunz, J. Appl. Phys. 101, 123110 (2007).

${ }^{9}$ A. Helbig, T. Kirchartz, R. Schaeffler, J. H. Werner, and U. Rau, Sol. Energy Mater. Sol. Cells 94, 979 (2010).

${ }^{10}$ T. Trupke, E. Pink, R. A. Bardos, and M. D. Abbott, Appl. Phys. Lett. 90, 093506 (2007).

${ }^{11}$ M. Glatthaar, J. Haunschild, R. Zeidler, M. Demant, J. Greulich, B. Michl, W. Warta, S. Rein, and R. Preu, J. Appl. Phys. 108, 014501 (2010).

${ }^{12}$ D. Hinken, K. Bothe, K. Ramspeck, S. Herlufsen, and R. Brendel, J. Appl. Phys. 105, 104516 (2009).

${ }^{13}$ A. Zekry and A. Al-Mazroo, IEEE Trans. Electron Devices ED 43, 691 (1996).

${ }^{14}$ P. O. Grabitz, U. Rau, and J. H. Werner, Phys. Status Solidi A 202, 2920 (2005).

${ }^{15}$ J. A. Giesecke, M. The, M. Kasemann, and W. Warta, Prog. Photovoltaics: Res. Appl. 17, 217 (2009).

${ }^{16}$ D. Hinken, C. Schinke, S. Herlufsen, A. Schmidt, K. Bothe, and R. Brendel, Rev. Sci. Instrum. 82, 033706 (2011).

${ }^{17}$ D. Hinken, K. Ramspeck, K. Bothe, B. Fischer, and R. Brendel, Appl. Phys. Lett. 91, 182104 (2007).

${ }^{18}$ M. Glatthaar, J. Haunschild, M. Kasemann, J. Giesecke, W. Warta, and S. Rein, Phys. Status Solidi RRL 4, 13 (2010).

${ }^{19}$ Y. Augarten, T. Trupke, M. Lenio, J. Bauer, J. W. Weber, M. Juhl, M. Kasemann, and O. Breitenstein, Prog. Photovoltaics: Res. Appl. 21, 933 (2013).

${ }^{20}$ T. Kirchartz, A. Helbig, and U. Rau, Sol. Energy Mater. Sol. Cells 92, 1621 (2008)

${ }^{21}$ J. Wong and M. A. Green, Phys. Rev. B 85, 235205 (2012).

${ }^{22}$ J. Wong and M. A. Green, IEEE Trans. Electron. Devices ED-58, 4011 (2011). 